\title{
The Modeling of Color Fatigue in 3-Dimensional Stereoscopic Video
}

\author{
Jeong-Yeop Kim, Sang-Hyun Kim, and Gil-Ja So
}

\begin{abstract}
The change of color information on 3-dimensional stereoscopic video usually causes visual fatigue. Color information also gives some effect on the depth recognition because of the visual perception for the effect such as 'forward color'. However the effect of 'forward color' does not gives much influence on depth perception. Therefore, the analysis of color itself rather than the concept of 'forward color' is important. In this paper, the method for calculating visual fatigue by color only is proposed.
\end{abstract}

Index Terms-Color fatigue, perceived depth, human visual system.

\section{INTRODUCTION}

In these days, 3-dimensional stereoscopic videos are very popular and can be seen in many places such as movie-theater, commercial complex, video cafe, display room of company, and so on. Especially, many people watches 3-d video at home with the help of 3-dimensional TV. Some people feel visual fatigue when they watch 3-dimensional video while others do not feel visual fatigue at all. Because the visual fatigue is related to personal physiological and psychological characteristic and it differs person to person. Therefore, it is important to make 3-dimensional videos safe to people watch them.

The concept of visual fatigue is researched by many people especially in making 3-dimensional commercial videos. In the process of making 3-D film and editing, the control of depth information is very important. There is no quantitative measure for this control because it depends on the personal characteristics of 3-D viewing. Therefore, it is difficult to make quantitative measure for representative cases.

However, there are some methods for describing the perceived depth information by using the parameters of 3-D viewing condition such as the observation distance from the display to the observer, inter-pupil distance of observer, device characteristic and so on. The characteristic of visual discomfort is one of the important issues in this field. Visual discomfort means the observer feels some fatigue with the specific observation parameters of observation environment such as observation angle and distance between the observer

Manuscript received November 30, 2014; revised March 2, 2015. This research is supported by Korea Creative Content Agency (KOCCA) in the Culture Technology(CT) Joint Research Center Project 2010.

Jeong-Yeop Kim is with University of Young-San, 99 Pilbong-gil Haeundae-Gu, Busan, 612-743, Korea (e-mail: neocopy@ysu.ac.kr).

Sang-Hyun Kim is with the Department of Computer Engineering, University of Young-San, 288 Junam-ro, Yangsan-si Kyungsangnam-do 626-790, Korea (e-mail: ksh50@ysu.ac.kr).

Gil-Ja So is with the Department of Cyber Police and Science, University of Young-San, 288 Junam-ro, Yangsan-si Kyungsangnam-do 626-790, Korea (e-mail: kjso@ysu.ac.kr). and display devices. There is visual discomfort zone [1], [2] and the observer feels visual fatigue when he or she watches 3-D contents [3], [4].

Visual discomfort zone means that if the observer is in discomfort zone when he or she observes 3-D videos on some display, he or she feels discomfort. In other case, when the observer is in comfort zone, he or she feels no discomfort for the 3-D videos. This phenomenon comes from the geometrical parameters such as the distance between the observer and the display, the inter-pupil distance of the observer, disparity of the image pixel position and dot pitch of display device and so on. This feeling of discomfort causes fatigue when the observer watches $3-\mathrm{D}$ videos. The fatigue does not caused from only these parameters mentioned above, however these parameters has potential influence on it.

Because there are so many other reasons for the observer feels fatigue. For example, the physical conditions, emotional changes, and so on. In the researches related the perception of depth assumes the fatigue comes from mainly from the geometrical parameters mentioned above and usually ignores those other reasons.

This is an important factor in the evaluation of perceived depth of observer. One of the methods includes depth tuning technique by defining safety zone. Chang Yuan [5] et al. calculated this kind of safety zone in some particular observation environment with fixed size of display device. They controlled the depth information by shifting and scaling based on human visual characteristics.

Kim et al. [6] tried to include the parameters from contents as color information into perceived depth model. They used color information as hue and lightness together. The perceived depth model was modified by using hue and lightness information in the form of scaling the depth.

In contrast to above approaches, the role of color information itself is more important than the effect of perceived depth by color parts. This is popular concept known as visual fatigue by color or color fatigue. The role of color parts of 3-dimensional stereoscopic videos is focused on the characteristic of color distribution only rather than the derived perceived depth by color. This color fatigue is represented on next chapter. In this paper, we are interested in the analysis of color fatigue and its parameters for the modeling of it. The method for making color fatigue model is proposed and tested in 3-dimensional stereoscopic videos.

\section{Conventional Methods}

\section{A. Conventional Perceived Depth Model}

Perceived depth model has been considered by many researchers and most of them did not take the effect of human 
visual factor and just thought about the algebraic structure of observation environment.

According to the research of Chang Yuan [5] et al., if the stereo videos are captured in parallel mode camera, the perceived depth is proportional to disparities and can be represented in (1) where, $D$ is perceived depth, $V$ is observation distance (distance from observer to display device), $I$ is IPD (inter-pupil distance) of observer, SD is horizon pixel pitch of display device and $d$ is disparity information.

$$
D=\frac{V}{\frac{I}{S_{D^{d}}}-1}
$$

The main parameters to decide visual comfort are observer-to-screen distance and disparity at screen. These two parameters have great influence on fatigue.

They shifted the images laterally to adjust disparity image and correct the data outside visual comfort zone. Additionally, if the shift values are not compatible for the scene, for example, very big to be shifted, these data can be scaled. By this approach, they can calibrate the 3-D images and make it comfortable when the observer watches the images. But there exist some artifact for disparity information because this approach adjusts the disparity values directly and there can be some discontinuity by this adjustment. In this paper, we are interested in some method how can we adjust the degree of depth not by direct adjustment of disparity.

Perceived depth model represents the actual depth perceived for some 3D images by human being in some observation condition. If we fix the observation condition such as observation distance, display device and so on, the perceived depth model gives constant depth information regardless of the displayed contents [5]. It is not considered the property of contents displayed on display device and the perceived depth is represented by just the physical, algebraic parameters and proportional rate of observation environment. However, according to the human visual experiment of depth perception [7], the actual perceived depth can be varied with the change of displayed contents such as wavelength or luminance values.

\section{B. Human Visual Experiment on Depth Perception}

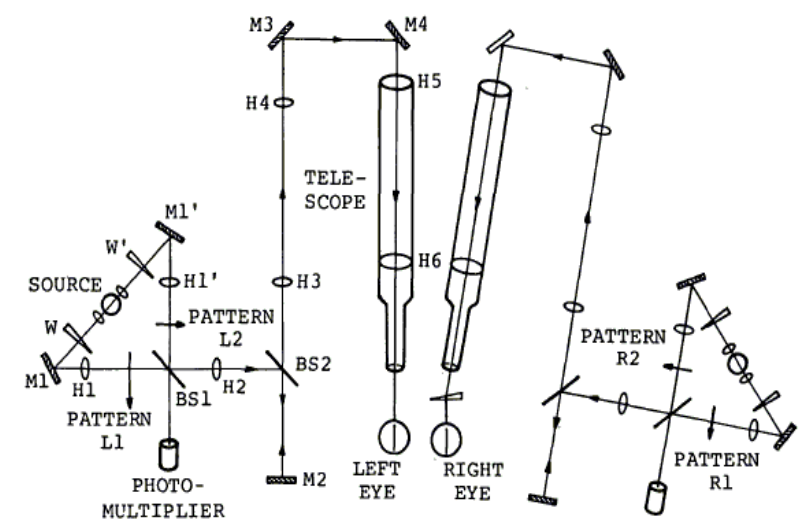

Fig, 1. Optical construction of experiments.

However, it is known that hue and luminance components give some influence on depth perception of human-being

from the physiological experimental reports about human vision and depth perception.

Cary Lu [7] et al. set experiment environment and generated stereopsis images. Stereopsis image by many type of combination was generated and used in experiment on if human being can recognize the depth information for the images or not. The contents of combination can be represented as wavelength $(\lambda)$ of patch luminance (L) information. These two factors can be manipulated by experimenter and therefore, gives many patterns for this experiment. The test environment is as shown in Fig. 1 and test pattern is shown in Fig. 2.

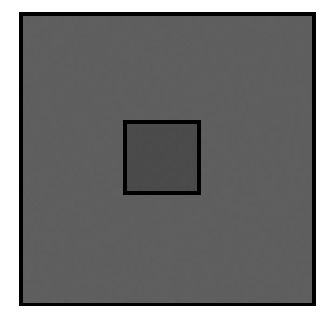

(a) left image

Fig. 2. Stereopsis pattern of optical image generation.

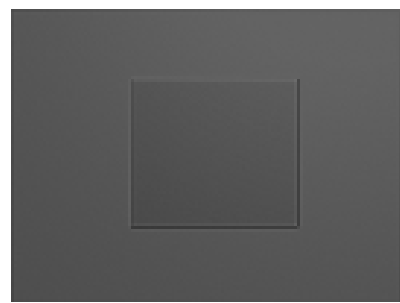

(a) left image

(b) right image

(b) right image
tern of Kim et al.

The result is shown in Table I and II. The depth of green object on red background is well perceived at low luminance (average contrast 10.5). The other case, red object should have high luminance (average contrast 1.5) to be perceived well. This fact of hysteresis means hue and luminance gives some influence on depth perception of human being.

TABLE I: RESULT OF CONTRAST: $\lambda_{1}=634 \mathrm{NM}, \lambda_{2}=512 \mathrm{NM}$

\begin{tabular}{ccc}
\hline \hline Patch1(red) & Patch2(green)1 & Contrast ratio(Patch 1/Patch 2)2 \\
\hline 256.47 & 26.40 & 9.78 \\
\hline 167.25 & 13.00 & 12.90 \\
\hline 104.25 & 8.95 & 11.68 \\
\hline 54.40 & 4.62 & 11.75 \\
\hline 34.21 & 3.86 & 8.80 \\
\hline 22.12 & 2.42 & 9.47 \\
\hline 13.08 & 1.42 & 9.17 \\
\hline
\end{tabular}

TABLE II: RESULT OF CONTRAST: $\lambda 1=512 \mathrm{NM}, \lambda 2=634 \mathrm{NM}$

\begin{tabular}{ccc}
\hline \hline Patch1(red) & Patch2(green)1 & Contrast ratio(Patch 1/Patch 2)2 \\
\hline 199.44 & 151.16 & 1.32 \\
\hline 97.36 & 45.38 & 2.17 \\
\hline 69.00 & 45.08 & 1.53 \\
\hline 45.03 & 31.33 & 1.45 \\
\hline 30.20 & 22.15 & 1.37 \\
\hline 19.30 & 15.03 & 1.27 \\
\hline 11.91 & 7.89 & 1.48 \\
\hline
\end{tabular}

\section{Perceived Depth Model with Color Information}

Kim et al. [6] included the parameters of color information into perceived depth model. They used color information as 
hue and lightness together. They proposed the perceived depth model as (2). The test pattern is shown in Fig. 3.

$$
D_{c}=\frac{V}{\frac{I}{s_{D^{d}}}-1} \times f\left(\lambda_{1}, L_{1}, \lambda_{2}, L_{2}\right)
$$

They concluded with (3) and showed the relationship between depth and wavelength, lightness.

$$
D_{c}=\frac{V}{\frac{I}{s_{D^{d}}}-1} \times \mathrm{a} \times\left(\frac{L_{1}}{0.0396 \lambda_{1}-0.0299 \lambda_{2}}-L_{2}\right)
$$

Their modeling was focused on the relationship between depth and color. However, the change of depth perception was not so dominant and it was difficult to test in real images.

\section{Color Fatigue}

There are many reasons for the color fatigue when people watch 3-dimensional videos. A. Bell [8] and D. Willard et al. [9] noticed that exposure to highly saturated colors can cause visual fatigue. They found visual fatigue would be observed when the eye has to continually refocus on different wave lengths of color. The cognitive decrease can be caused by the subjects being unable to process the plot or other key elements as the brain is struggling to follow the rapid and various visual refocus phenomenon.

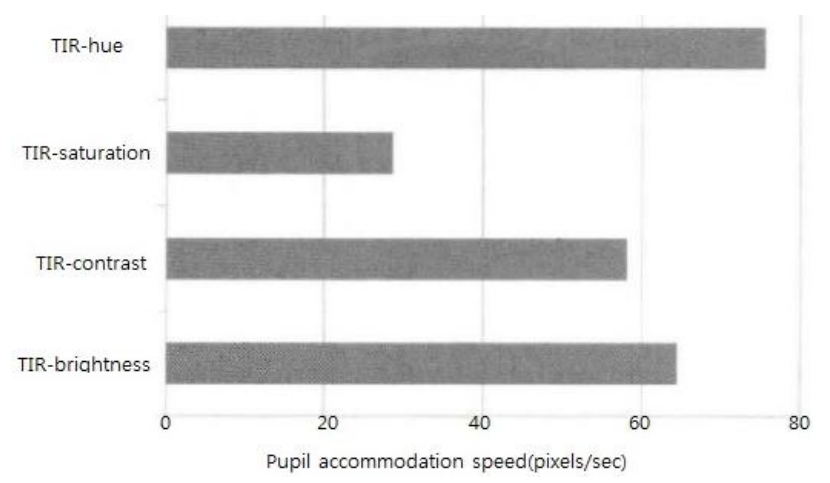

Fig. 4. Comparing pupil accommodation speeds.

A. Bell [8] also described relationship between violence level of some prison and colors of environment. They found the fact that the level of violence increased in red and yellow painted wings, rather than the blue and green wings. While pink was found to have a tranquilizing and calming effect, suppressing prisoner hostility and aggressiveness by reducing energy levels. For the color of red light, it was believed to increase strength by $13.5 \%$ and can be used for athletes to increase their athletic performance when they need short, quick bursts of energy. And blue light assists those athletes for steady energy output. They concluded bright yellow was the most eye sensitive color, and it causes hostility and aggressiveness. They also suggested that the use of yellow high pressure sodium street lighting may not be appropriate for exterior lighting.

Y. J. Kim et al. [10] focused on the effect of hue changes by the analysis of brainwave to measure visual fatigue. They used LCD screen and changed the video characteristic such as hue, brightness, saturation and contrast. The measure for the visual fatigue was frequency of eye blink and pupil accommodation speeds. Among the above 4 parameter changes, the most effective factor was hue change and the next rank was brightness, contrast and saturation in series. The result is shown in Fig. 4.

P. E. Nobbs [11] focused the color changes especially on successive contrast. A visual process usually called fatigue plays a large part in such matters, the eye that has been fixed upon an area of saturated color tending, on sudden removal, to convey the impression of a complementary, or negative, after-image. He found the cause of fatigue as the eye does not fix and by constant movement renews the retinal apparatus of registration. Therefore, it is important to check the change from saturated color to grey components for the analysis of visual fatigue.

E. Goldstein [12] described about the long lasting color region in the video without any change in some time period. He noticed that people have a red-green ganglion cell in the retina whose baseline activity is rather weak when they expose it to white light. However, the cell's activity is increased by red light, so they experience the color red. If the stimulus of red light is input to a specific cell for a long enough period of time, the cell becomes fatigued. If we then swap out the red light with white light, the now-tired cell responds even less than the original baseline. This fact means the stimulus for a long time causes the sensitivity of cell more insensitive and causes visual fatigue.

E. Keyes [13] noticed that color can create "a visual layer that we separate perceptually" from other information. In his conclusion, numerous researchers and authors have suggested using color to highlight salient features, color code related bits of information, decreased the cognitive load, and simplify complex information. The color of text can cause additional fatigue compared to grey cases.

J. Carmody et al. [14] found excessive color contrast above $70 \%$ can cause visual fatigue.

R. Rider [15] noticed the cold hue can cause decrease of blood pressure therefore decrease visual fatigue.

In this section, many attributes of color related to the visual fatigue were reviewed and some of these attributes would be used in fatigue modeling in chapter IV.

\section{EXPERIMENTAL ENVIRONMENT}

The test videos for this new model can be obtained from the released advertisement videos by display device companies such as LG, Samsung and so on. The 6 videos were selected as input and shown in Fig. 5.

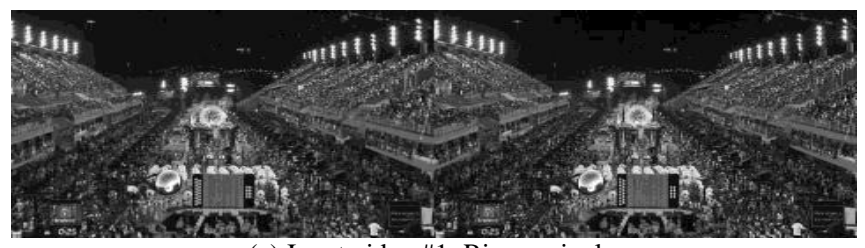

(a) Input video \#1: Rio-carnival.

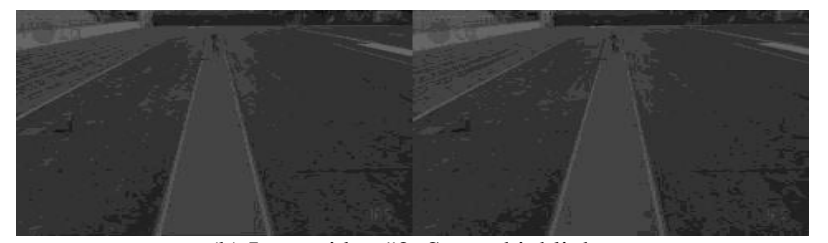

(b) Input video \#2: Sports highlight. 


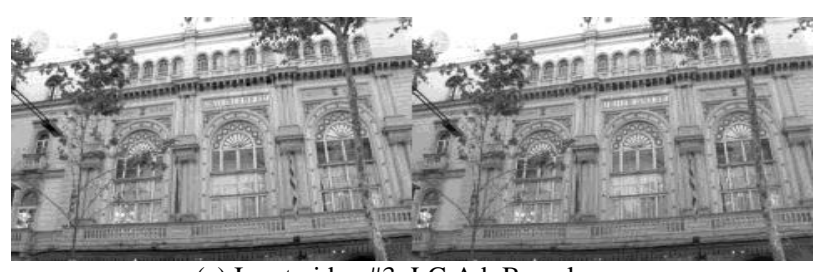

(c) Input video \#3: LG Ad. Barcelona.

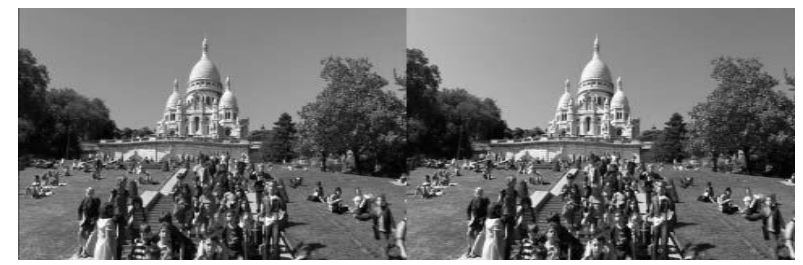

(d) Input video \#4: LG Ad. Paris.

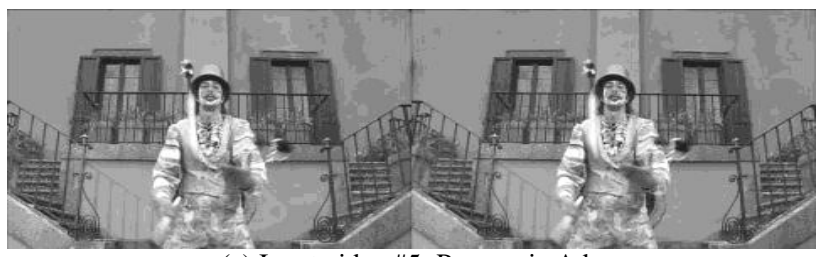

(e) Input video \#5: Panasonic Ad.

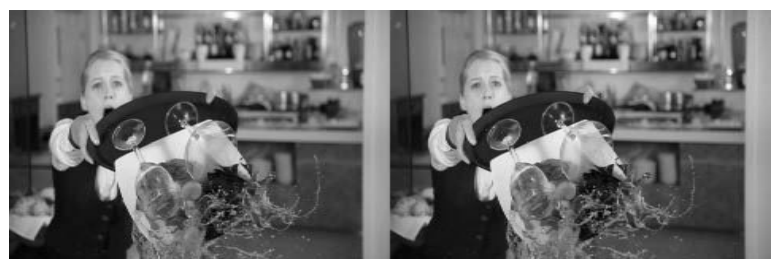

(f) Input video \#6: Samsung Ad.

Fig. 5. Input 3-dimensional stereoscopic videos.

All the selected videos from \#1 to \#6 contain vivid colorful object or people with colorful clothes for the test of visual fatigue. The size of input videos and related information are shown in Table III.

TABLE III: INFORMATION OF INPUT VIDEOS

\begin{tabular}{ccccc}
\hline $\begin{array}{c}\text { Input } \\
\text { number }\end{array}$ & $\begin{array}{c}\text { Total } \\
\text { frames }\end{array}$ & contents & $\begin{array}{c}\text { Running } \\
\text { time }\end{array}$ & Video size \\
\hline$\# 1$ & 6370 & Rio-Carnival & $4: 38$ & $1920 \times 1080$ \\
\hline$\# 2$ & 2800 & Sports Highlight & $2: 16$ & $1920 \times 1080$ \\
\hline$\# 3$ & 3326 & LG Ad. Barcelona & $2: 44$ & $1920 \times 1080$ \\
\hline$\# 4$ & 4000 & LG Ad. Paris & $3: 49$ & $1920 \times 1080$ \\
\hline$\# 5$ & 4440 & Panasonic Ad. & $3: 13$ & $1920 \times 1080$ \\
\hline$\# 6$ & 3743 & Samsung Ad. & $2: 41$ & $1920 \times 1080$ \\
\hline
\end{tabular}

\section{PROPOSED MODELING}

In this paper, the factors mentioned above chapter II were reviewed and some of them are chosen for the modeling of color fatigue. For the easy and fast calculation of parameters for the model, the input video frame is divided into $N \times N$ regions. The parameters for color fatigue would be calculated by each block of regions and be integrated into unique values for visual color fatigue.

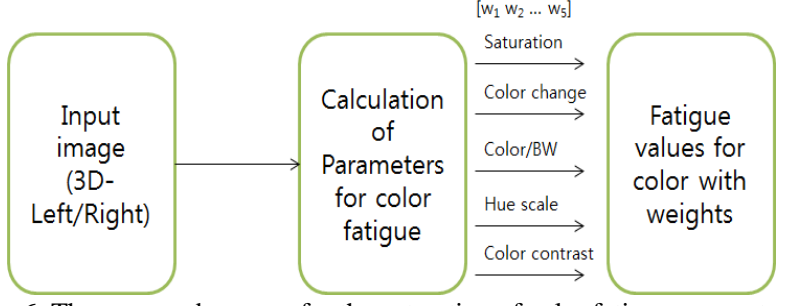

Fig. 6. The proposed process for the extraction of color fatigue parameters.
The calculation of parameters can be performed by whole frame. If there are some local changes, the global parameter values would be very low. However, the small local change can introduce big stimulus to the observer. Therefore, we used block scheme for the calculation of visual fatigue. The flow of proposed model is shown is Fig. 6 and divided frame is shown in Fig. 7.

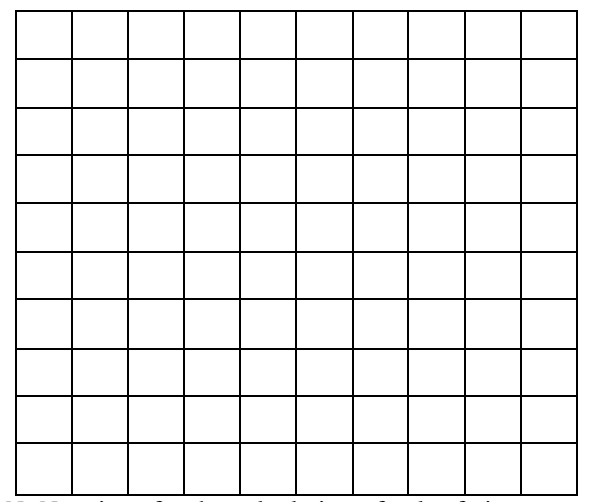

Fig. 7. $N \times N$ regions for the calculation of color fatigue parameters.

The scheme for the divided image in Fig. 7 is applied into whole frames in each test video. If some parameters need comparison between frames, the corresponding block would be used.

The selected parameters from chapter II are as follows:

1) The number of blocks $\left(N_{S}\right)$ in one frame with some excessive saturations with some threshold

2) The number of blocks $\left(N_{H}\right)$ with comparatively big $\mathrm{dE}$

$$
\mathrm{dE}=\frac{1}{N} \sum_{i=1}^{N}\left[\left(C_{1, f+1}-C_{1, f}\right)^{2}+\left(C_{2, f+1}-C_{2, f}\right)^{2}\right]
$$

where $f$ is frame number and $C_{1}, C_{2}$ are hue vectors of block color for each frame.

3) The presence of change in color and grey components.

There is a color frame and the successive block in time is grey. The calculation parameter is as follows $\left(N_{G}\right)$ :

$$
20-\frac{s_{f}}{S_{f+1}}
$$

where $S$ is saturation and $f$ is frame number in time. If this value is big, it means there is a change from color to grey.

4) Hue scale $\left(H_{S}\right)$

This parameter is related to the value of hue than causes visual fatigue in specific case.

yellow: 4 , green: 3 , cyan: 2 , blue: 1 , magenta: 0 , red: 1

5) The number of blocks with big color contrast $\left(N_{C}\right)$.

For the color contrast, there are 2 ways of calculation. In this paper, the variance of color in each block is used for the color contrast.

Therefore, by integrating the above 5 specific parameters the model for color fatigue of stereoscopic video can be obtained. The visual fatigue $F_{C}$ cased only by color is as follows:

$F_{C}=w_{1} \times N_{S}+w_{2} \times N_{H}+w_{3} \times N_{G}+w_{4} \times N_{C}+w_{5} \times H_{S}$

The visual fatigue by color can be calculated by the above model for each frame and can be threshold properly. 
The input stereoscopic videos of Fig. 5 are used for the calculation and the result is shown in Table IV.

TABLE IV: FORWARD MATCH POINTS(FRAME NUMBER)

\begin{tabular}{c|c|c|c|}
\hline Video & $\begin{array}{c}\text { Total average - } \\
F_{c}\end{array}$ & $\begin{array}{c}\text { Number of frames } \\
\text { exceeds threshold }\end{array}$ & $\begin{array}{c}\text { Frequency of frames } \\
\text { exceeds threshold } \\
{[\%]}\end{array}$ \\
\hline Video \#1 & 1.560 & 130 & 2.041 \\
\hline Video \#2 & 2.131 & 223 & 7.970 \\
\hline Video \#3 & 2.037 & 75 & 2.256 \\
\hline Video \#4 & 1.894 & 72 & 1.800 \\
\hline Video \#5 & 1.764 & 167 & 3.762 \\
\hline Video \#6 & 1.673 & 109 & 2.913 \\
\hline \hline Average & 1.843 & & 3.457 \\
\hline
\end{tabular}

The average play time of each video is about 2 minute and 30 frames for each second. The calculation weight in equation (6) was set to 0.2 for each. The resultant fatigue values ranged from 0 to 3.0 or so as shown in Fig. 8. The horizontal axis represents frame number and vertical axis is fatigue value.

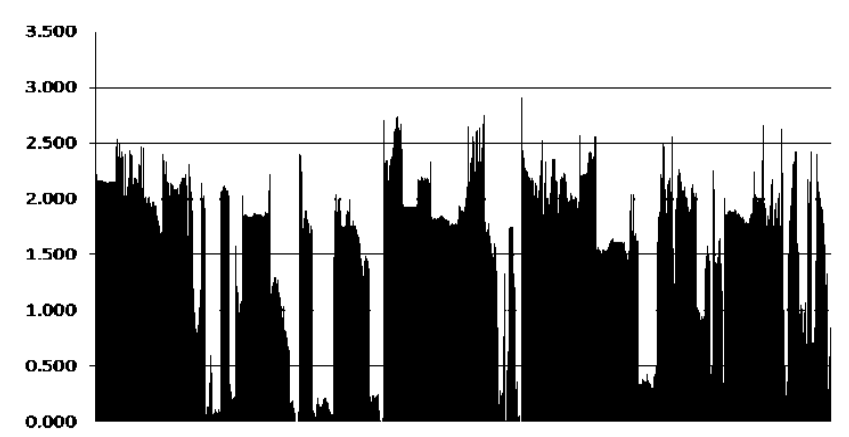

Fig. 8. The result of color fatigue parameters for video \#1.

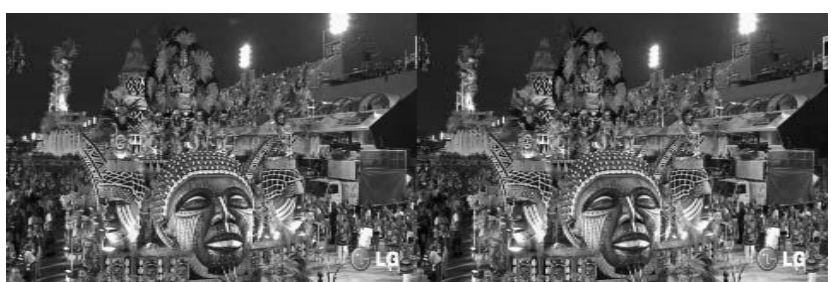

(a) Input video \#1: Rio-carnival $(f: 1001)$.

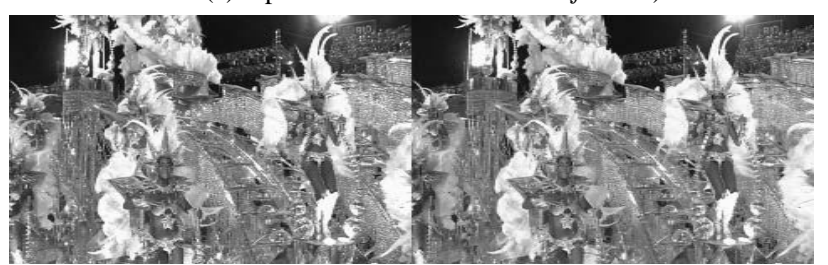

(b) Input video \#1: Rio-carnival(f: 3714).

Fig. 9. Sample frame for very low and excessive color fatigue $F_{c}$ for input video \#1.

In Table IV, the change of fatigue value for video \#2 and \#3 is bigger than other input videos. This means video \#2 and \#3 gives more fatigue than any other videos when observed. To check out the effect of $F_{C}$, two frames of test video \#1 are extracted and shown in Fig. 9. The value of $F_{C}$ in frame number 1001 was 0.055 and shown in (a) and 2.918 for frame number 3714 . The frame of (a) has not much change of color components, however, frame of (b) was at the point of scene change and therefore gives much change of color components. By this example, our model of $F_{C}$ can be thought as reasonable for the calculation of color fatigue. Other frames of input for high color fatigue are shown in Fig.
10.

Most frames of Fig. 10 corresponds scene change frames and therefore gives high visual color fatigue.

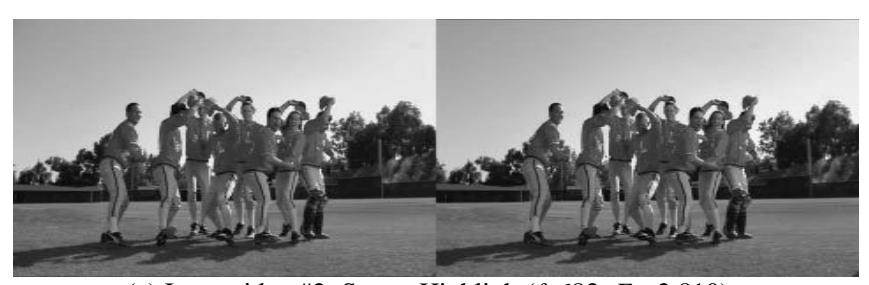

(a) Input video \#2: Sports Highlight $\left(f: 683 ; F_{C}: 2.810\right)$.

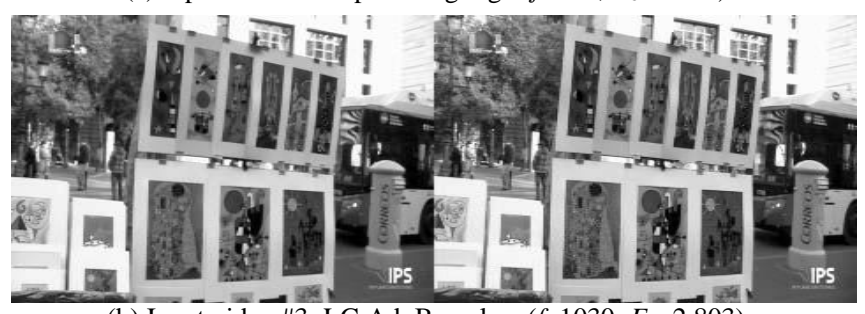

(b) Input video \#3: LG Ad. Barcelona $\left(f: 1030 ; F_{C}: 2.803\right)$.

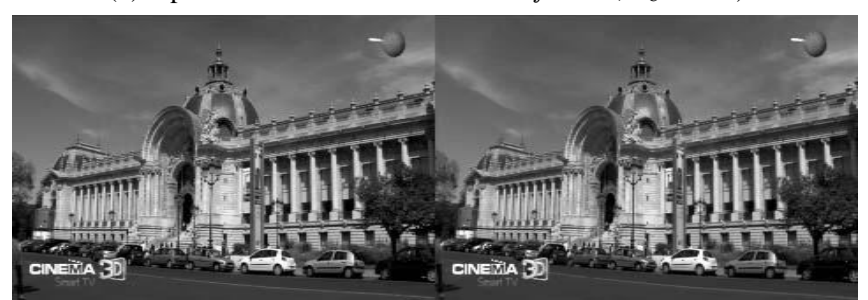

(c) Input video \#4: LG Ad. Paris(f: 1952; $\left.F_{C}: 2.774\right)$.

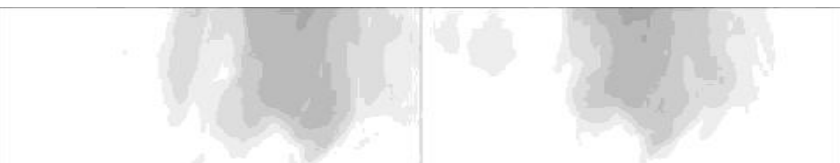

(d) Input video \#5: Panasonic Ad.(f: 2640; $\left.F_{C}: 3.343\right)$.

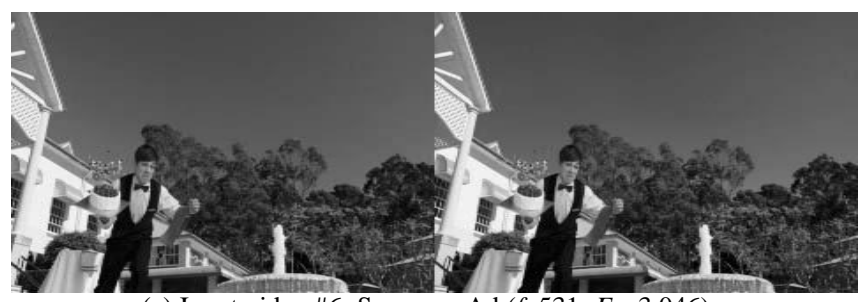

(e) Input video \#6: Samsung Ad.(f: 531; $F_{C}$ : 3.046).

Fig. 10. Sample frame for excessive color fatigue $F_{c}$ for other input video.

\section{CONCLUSION}

In this paper, the method for finding relationship between the visual fatigue and change of color information is proposed in the form of color fatigue model. By the proposed method, we found the correct video frames that give much fatigue when people watch them. This model contains color characteristics such as saturation, color variance, color to grey change, hue and color contrast. In the experiment, the average percentage of frames with excessive color fatigue was $3.457 \%$ for all 6 test videos. Test video \#2 was the worst case in the view of color fatigue as $7.97 \%$, while video \#3 gives $1.8 \%$ as the best case.

The color fatigue model only counts color component in the test video, this model can be utilized in the integration stage of general visual fatigue model as a part of color matters. The proper decision of actual threshold for the color fatigue value $F_{C}$ is needed in the further studies. 


\section{ACKNOWLEDGMENT}

This work was supported by the ICT R\&D program of MSIP/IITP. [2014(15501-14-1013), 3D Stereoscopic Image Production Research and Development].

\section{REFERENCES}

[1] L. Marc, F. Marten, H. Ingrid, and I. Wijnand, "Visual discomfort and visual fatigue of stereoscopic displays: A review," Journal of Imaging Science and Technology, vol. 53, no. 3, pp. 30201-1-30201-14, May 2009.

[2] S. Lee, Y. Jung, H. Sohn, Y. Ro, and H. Park, "Visual discomfort induced by fast salient object motion in stereoscopic video," in Proc. SPIE 7863, Stereoscopic Displays and Applications XXII, February 11, 2011, p. 786305.

[3] I. P. Howard and B. J. Rogers, Binocular Vision and Stereopsis, 1st ed. Oxford University Press, 1995.

[4] D. M. Hoffman, A. R. Girshick, K. Akeley, and M. S. Banks, "Vergence-accommodation conflicts hinder visual performance and cause visual fatigue," Journal of Vision, vol. 8, no. 3, pp. 1-30, 2008.

[5] C. Yuan, H. Pan, and S. Daly, "Stereoscopic 3D Content depth tuning guided by human visual models," in Proc. SID Symposium Digest of Technical Papers, June 2011, vol. 42, issue 1, pp. 916-919.

[6] J. Kim, S. Kim, and G. So, "Perceived depth modeling based on color information," International Journal of Computer Theory and Engineering, vol. 5, no. 4, pp. 716-720, 2013.

[7] C. Lu and D. H. Fender, "The interaction of color and luminance in stereoscopic vision," Investigative Ophthalmology, pp. 484-490, June 1972.

[8] A. Bell, "The effect of colour on audience response in theatre scenic design," Thesis for the honours degree in theatre and drama studies, Murdoch University, 2013.

[9] D. Willard, J. E. Cobble, and S. J. Gertel. (2008). Color in an optimum learning environment. International Center for Leadership in Education. [Online]. Available: www.leadered.com/pdf/Colorwhitepaper.pdf

[10] Y. Kim, E. Lee, M. Whang, and K. Park, "A survey of objective measurement of fatigue caused by visual stimuli," Journal of the Ergonomics Society of Korea, vol. 30, no. 1, pp. 195-202, February 2011.

[11] P. E. Nobbs, A Treatise in the Discovery of Form, Oxford University Press, London New York Toronto, 1937, ch. 5.

[12] E. Goldstein, "Sensation and perception," Cengage Learning, ch. 3, 2013.

[13] K. Elizabeth, "Typography, color, and information structure," Technical Communication, vol. 40, pp. 638-638, 1993.

[14] J. Carmody and W. Trusty, "Life cycle assessment tools, implication," A Newsletter by Informe Design, vol. 5, issue 3, 2005.

[15] R. Rider, "Color psychology and graphic design applications," Thesis for the honors program, Liberty University, Spring 2009.

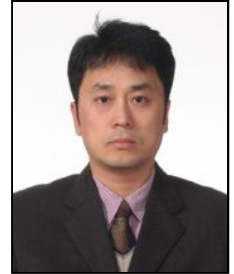

Jeong-Yeop Kim received the diploma in electronics engineering from Kyung-Pook National University of Daegu, South Korea in 1990; M.S. and PhD degrees both in image processing of electronics engineering, from the same university, in 1992 and 2000, respectively.

He was with the Samsung Advanced Institute of Technology in Korea as a senior research staff from 1994 to 2000. From March 2001 to February 2013, he was with the Department of Game Contents Science of the University of Young-San at Busan, as an associate professor. From March 2013, He is with the Department of Undeclared Major of the University of Young-San at Busan. His doctoral dissertation work focused on detecting and adjusting the white point from single color image. The topics he has worked on include color printing, error diffusion, color correction and 3D stereoscopic images. His current research is on correction of color data in 3D stereoscopic images.

$\mathrm{He}$ is a member of the KMMS (Korea Multimedia Society) and KIPS (Korea Information Processing Society).

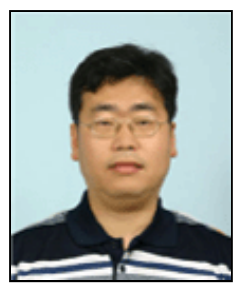

Sang-Hyun Kim received the diploma in electronics engineering from Kyung-Pook National University of Daegu, South Korea in 1991; M.S. and PhD degrees both in image processing of electronics engineering, from the same university, in 1993 and 1998, respectively.

He was with the Samsung Medison Co. Ltd, in Korea as a senior researcher from 1998 to 2001. From September 2001, he is with the Department of Computer Engineering of the University of Young-San at Yangsan, Kyungnam as an associate professor. His doctoral dissertation work focused on the development of fractal image coding method and transmission for image processing, image communication and computer vision problems. The topics he has worked on include virtual reality, ultrasound image processing, medical image processing, image feature extraction and classification, and 3D stereoscopic images. His current research is on region-based intensity correction in 3D stereoscopic images.

$\mathrm{He}$ is a member of the KMMS (Korea Multimedia Society) and KIPS (Korea Information Processing Society).

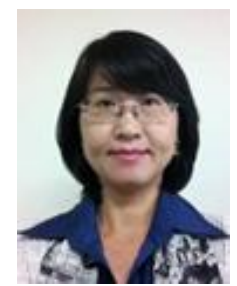

Gil-Ja So received the diploma in computer science from the Dong-Eui University of Pusan, South Korea in 1994; and M.S. and $\mathrm{PhD}$ degrees both in natural language processing of computer engineering, from the Pusan National university, South Korea in 1997 and 2012, respectively. From September 2001, she is with the Department of Computer Engineering of the University of Young-San at Yangsan, Kyungnam as an lecturer. Her doctoral dissertation work focused on the Korean grammar checker for parsing Korean documents and correcting context-sensitive words in documents. The topics she has worked on include virtual reality, game development and artificial intelligence of non-players of game, and 3D stereoscopic images. Her current research is on region-based intensity correction in 3D stereoscopic images.

She is a member of the KMMS (Korea Multimedia Society) and KIPS (Korea Information Processing Society). 